\title{
Efectos del programa de prevención Saluda: una revisión de estudios controlados de evaluación
}

\author{
José P. Espada Sánchez ${ }^{1}$ y Olga Hernández Serrano ${ }^{2}$ \\ ${ }^{1}$ Departamento de Psicología de la Salud, Universidad Miguel Hernández, Elche \\ ${ }^{2}$ Departamento de Fisioterapia, Escola Universitària de la Salut i l'Esport. \\ Universitat de Girona, Girona
}

\section{España}

Correspondencia: Dra. Olga Hernández Serrano. Escola Universitària de la Salut i l'Esport. Universitat de Girona. Departamento de Fisioterapia. Campus de Salt. Carrer Francesc Macià, 65 - 17190. Salt (Girona). España. E-mail: olga.hernandez@cadscrits.udg.edu; ohernandez@euses.cat 


\section{Resumen}

El objetivo del presente estudio es revisar las evidencias sobre la eficacia del programa Saluda, un protocolo de prevención escolar del consumo de drogas dirigido a adolescentes. Se describen los contenidos del programa y se revisan los resultados de nueve ensayos controlados que evaluaron los efectos del programa. Los participantes fueron estudiantes españoles con edades de 12 a 16 años. El programa fue elaborado a partir de los resultados de una revisión cuantitativa de programas españoles, donde se identificaron los componentes más eficaces. Los primeros ensayos compararon sus resultados respecto a un programa de eficacia probada y una intervención inespecífica de promoción de la salud, mostrando efectos positivos. Estudios posteriores evaluaron los efectos del programa según el tipo de aplicador, la edad de los participantes, y los efectos sobre aspectos actitudinales y cognitivos. Los estudios más recientes se han centrado en analizar la eficacia de los componentes del programa y la contribución de las tareas asignadas. En este estudio se sintetizan las evidencias empíricas sobre los efectos del programa para modificar variables de riesgo y protección y se plantean líneas de futuro para mejorar la eficacia y eficiencia del programa.

Palabras Clave: abuso de drogas, prevención escolar, adolescencia, programa de prevención. 


\title{
Effects of the Saluda prevention program: a review of controlled evaluation studies
}

\begin{abstract}
The objective of the present study is to review the evidence on the effectiveness concerning the Saluda program, a school-based substance use prevention protocol used amongst adolescents. We provide a description of the program's content and the results from nine controlled trials evaluating the program's effectiveness. Participants were Spanish students aged between 12 and 16 years old. The program was created on the basis of the results from a quantitative review focused on Spanish programs, in which the most effective components were identified. Early trials compared their results with respect to a proven-effectiveness program and an unspecific health-promotion intervention, showing positive effects. Subsequent studies focused on the effects of the program according to the type of applicant, participants' age, and attitudinal and cognitive aspects. Recent studies focused on analysing the programcomponents effectiveness, as well as the contribution of the assigned tasks. This study summarizes strong empiric evidence concerning the program effectiveness on both risk and protective variables, at the time that further lines to improve the program's effectiveness and efficiency are established.
\end{abstract}

Keywords: drug abuse, school-based prevention, adolescence, prevention program.

Reception: 10/20/14 Initial acceptance: 11/25/14 Final acceptance: 03/03/15 


\section{Introducción}

La adolescencia es la etapa donde típicamente se produce el inicio del consumo de drogas por la mayor presencia de factores de riesgo (Sussman, Unger y Dent, 2004). El Observatorio Español de la Droga y las Toxicomanías (OEDT, 2013) indica que el alcohol, el tabaco y el cannabis son las sustancias más consumidas entre los escolares de 14 a 18 años, con un $83.9 \%, 43.8 \%$, y $33.6 \%$ de consumidores durante el último año, respectivamente. En la actualidad el alcohol en España sigue vinculado al ocio ya que es durante el fin de semana cuando el consumo es más extenso. Aunque se ha intensificado la puesta en marcha de actuaciones preventivas, el consumo de sustancias se inicia antes de los 14 años en el caso de las drogas legales, por ejemplo alcohol y tabaco, y una iniciación más tardía (15 años cumplidos) en el caso de sustancias como cocaína, éxtasis, anfetaminas o alucinógenos (OEDT, 2013).

Datos recientes en población adolescente española indican que el 64\% de los encuestados declara haber tenido una intoxicación aguda en el último año (González, 2014). El patrón de consumo abusivo incluye posibles interferencias en el desarrollo neurológico, la memoria, el aprendizaje y la planificación de tareas (Brown, 2008; Masten y Faden, 2008). El atracón de alcohol se ha asociado a comportamientos problemáticos como el absentismo escolar, desvinculación con la escuela, violencia sexual y de pareja, o mayor riesgo de abusar del alcohol (Miller, Naimi, Brewer y Everett, 2007).

Ante esta situación, continúa existiendo la necesidad de contar con programas eficaces de prevención del consumo de sustancias. Los estudios de revisión sistemática ofrecen evidencias de la eficacia de los programas de prevención escolar para reducir el consumo de sustancias (Faggiano et al., 2008; Karki et al., 2012). No obstante, a pesar de los esfuerzos por reducir el consumo, el impacto de los programas es todavía limitado. Muchas de estas intervenciones presentan un pequeño tamaño del efecto (Espada, Gonzálvez, Orgilés, Lloret y Guillén-Riquelme, 2015) y encuentran dificultades para mantener en el tiempo su impacto (Canning, Millward, Raj y Warm, 2004; Gázquez, García y Espada, 2009).

Teniendo en cuenta los elementos que determinan la eficacia de los programas, y al margen de la importancia que supone valorar su efecto global, algunos estudios de revisión sistemática (Karki et al., 2012) y meta-análisis (ej. Tobler et al., 2000) han tratado de identificar qué ingredientes activos son responsables de los efectos de las intervenciones. Otra estra- 
tegia para conocer estos elementos consiste en la manipulación experimental de los componentes de la intervención a través de un desmantelamiento total o parcial del programa. Sin embargo, en España son todavía escasos los estudios que lleven a cabo desmantelamientos de programas.

El tipo de agente responsable de la implementación del programa es otro aspecto que puede influir de forma importante en los resultados de las actuaciones preventivas. Unos autores opinan que el profesorado presenta suficiente conocimiento en los contenidos (Hunter y Elias, 2000), y otros perciben al agente externo como un experto en la temática (Espada, Rosa y Méndez, 2003). Desde una perspectiva más práctica, hay quienes indican la necesidad de analizar las competencias que debe poseer un monitor eficaz (Cuijpers, 2002).

\section{Objetivo}

La principal fuente de información para mejorar los programas preventivos son los resultados de los estudios sobre la eficacia y la eficiencia de las intervenciones. De este modo es posible discernir entre aquellos aspectos que funcionan y los que no lo hacen, mejorando así la relación coste/beneficio de las intervenciones. Este trabajo revisa los estudios sobre el Programa Saluda (Espada y Méndez, 2003) que mediante nueve ensayos controlados llevados a cabo durante doce años ha sido puesto a prueba en población adolescente en España. El objetivo de este trabajo es describir el desarrollo del programa y presentar una síntesis de las investigaciones que han contrastado su eficacia, junto con futuras líneas de trabajo.

\section{Programa Saluda}

\section{Desarrollo del programa}

El programa Saluda (Espada y Méndez, 2003) se centra en la prevención del abuso de alcohol y otras drogas recreativas en el ámbito del ocio. Está destinado a escolares entre 12 y 14 años que no se han iniciado en el consumo o que se encuentran en fases iniciales, ya que se enmarca entre los programas de prevención primaria. Se clasifica entre los programas de desarrollo de competencias, y teóricamente se basa en el Modelo del Aprendizaje Social y en la Teoría de la Acción Razonada. Los objetivos del programa son retrasar la edad de inicio al consumo de alcohol y reducir el consumo abusivo de alcohol y otras drogas especialmente en el contexto del fin de semana. En la tabla 1 se presenta una síntesis de las diez sesiones de las 
que consta el programa con sus respectivas fases y algunos ejemplos de actividades (grupales/individuales).

Tabla 1. Programa Saluda: fases, estructura general de las sesiones y ejemplos de actividades

\begin{tabular}{|c|c|c|}
\hline Fase & Sesión & Ejemplos de actividades \\
\hline \multirow[b]{2}{*}{ Educativa } & 1. Información general & $\begin{array}{l}\text { Actividad individual y grupal: } \\
<<\text { Clasificando las drogas }>>\end{array}$ \\
\hline & 2. Información sobre factores de riesgo & $\begin{array}{l}\text { Actividad grupal: }\langle<\text { Factores de } \\
\text { riesgo del abuso de alcohol y del } \\
\text { consumo de drogas de síntesis }>>\end{array}$ \\
\hline \multirow{7}{*}{$\begin{array}{l}\text { Entrenamiento en } \\
\text { Habilidades }\end{array}$} & 3. Percepción normativa & $\begin{array}{l}\text { Actividad grupal: }<<\text { Percepción } \\
\text { normativa }>>\end{array}$ \\
\hline & 4. Influencia de la publicidad & $\begin{array}{l}\text { Actividad individual: }<<\text { Análisis } \\
\text { de anuncios publicitarios }>>\end{array}$ \\
\hline & 5. Actividades de ocio saludable & $\begin{array}{l}\text { Actividad individual: }<<\text { ¿A qué } \\
\text { dedico mi tiempo?>> }\end{array}$ \\
\hline & 6. Habilidades sociales generales & $\begin{array}{l}\text { Actividad individual: }<<\text { Q Quién es } \\
\text { quién?: el bueno, el feo y el ma- } \\
\text { lo〉> }\end{array}$ \\
\hline & $\begin{array}{l}\text { 7. Habilidades sociales centradas en el } \\
\text { consumo de sustancias }\end{array}$ & $\begin{array}{l}\text { Actividad grupal: }<<\text { Escenifica- } \\
\text { ciones: habilidad para decir no >> }\end{array}$ \\
\hline & 8. Resolución de problemas generales & $\begin{array}{l}\text { Actividad grupal: }<<\text { El método de } \\
\text { resolución de problemas }>>\end{array}$ \\
\hline & $\begin{array}{l}\text { 9. Resolución de problemas centrados en } \\
\text { el consumo de sustancias }\end{array}$ & $\begin{array}{l}\text { Actividad individual: }<<\text { Tomando } \\
\text { decisiones }>>\end{array}$ \\
\hline Mantenimiento & 10. Estrategias de mantenimiento & $\begin{array}{l}\text { Actividad individual: }<<\text { Compro- } \\
\text { miso público: consumo sí/consumo } \\
\text { no >> }\end{array}$ \\
\hline
\end{tabular}

\section{Estudios iniciales}

A partir de los resultados de un meta-análisis sobre la eficacia de los programas para prevenir el abuso de drogas aplicados en el contexto escolar en España entre 1985 y 2002 (Espada et al., 2002), se seleccionaron los componentes más eficaces, junto a un análisis de las nuevas tendencias de consumo. Con la versión piloto del programa se llevó a cabo un estudio que incluyó los componentes que habían mostrado mayores resultados positivos. La versión preliminar del programa se evaluó en 2001 con el nombre de Saluda al fin de semana y respondió al reto de enfrentarse a los nuevos patrones de consumo juvenil de sustancias como la moda del botellón y el consumo de pastillas. Se pretendía ofrecer datos sobre la relación de los factores de riesgo del consumo de alcohol y las drogas sintéticas, y especialmente sobre los efectos preventivos del programa. Este estudio aportó los primeros datos sobre la eficacia del programa hallando resultados positivos para modificar significativamente las variables mediadoras del consumo específicas e inespecíficas. 
Efectos del programa sobre factores cognitivos

Espada, Orgilés, Méndez, García-Fernández e Inglés (2008) realizaron un análisis sobre los efectos del programa Saluda en relación a tres factores de riesgo de tipo cognitivo: conocimientos sobre las drogas, percepción de riesgo y estimación normativa del consumo. Los participantes fueron estudiantes de tercer curso pertenecientes a un centro de Educación Secundaria. Cada uno de los grupos (aulas de clase) fueron asignados aleatoriamente a una de las cinco condiciones siguientes: programa Saluda, intervención placebo, intervención inespecífica (programa de Educación para la Salud), un programa de eficacia probada (Life Skills Training) y un grupo control que no recibió intervención. Se utilizó un diseño cuasiexperimental con mediciones pretest, postest y seguimiento (tras 3 y 6 meses de su aplicación postest).

Se encontró que las intervenciones resultaron más eficaces que la no intervención para incidir sobre variables protectoras cognitivas. Comparando los dos tratamientos principales entre sí (Saluda y Life Skills Training), ambos consiguieron mejorar las capacidades cognitivas de protección frente al consumo de drogas. Sin embargo, cuando ambos se compararon con el programa de Educación para la salud se observó que los programas específicos para prevenir el abuso de sustancias producían cambios mayores sobre las variables controladas. Al mismo tiempo, Saluda se mostró más eficaz para incidir sobre el nivel de conocimientos de alcohol y drogas sintéticas que el resto de intervenciones y la no-intervención.

El análisis comparativo de los tres programas activos con la atención placebo mostró resultados superiores en las tres intervenciones para disminuir la intención de consumo. Sin embargo, el programa Saluda fue el más eficaz para incrementar la percepción del riesgo de consumo de alcohol tanto en la evaluación postest, tras la intervención, como en el seguimiento a los 3 meses y 6 meses.

\section{Eficacia según la edad de los participantes}

Pereira y García-Fernández (2009) llevaron a cabo un estudio sobre los efectos del programa Saluda según la edad de los participantes. La muestra estuvo compuesta por estudiantes pertenecientes a dos centros de Educación Secundaria. Mediante un diseño con dos condiciones experimentales (grupo 1: adolescencia temprana de 11 a 13 años, y grupo 2: adolescencia media de 14 a 16 años) y dos grupos control sin intervención (grupo 3: adolescencia 
temprana de 11 a 13 años y grupo 4: adolescencia media de 14 a 16 años). Para ello, se llevó a cabo un muestreo aleatorio estratificado donde los distintos estratos estaban compuestos por aproximadamente el mismo número de participantes. El programa se mostró eficaz para disminuir la frecuencia del consumo de alcohol, la norma percibida y la intención de consumo, siendo mayor este impacto en la adolescencia temprana para todas estas variables. Sin embargo, no se hallaron efectos positivos cuando se comparó los grupos que recibieron intervención frente a los grupos control para incidir sobre la percepción de riesgo del consumo de sustancias.

\section{Eficacia según el agente de implementación}

Gázquez (2010) analizó los efectos del programa en función del agente aplicador del programa. Para ello, el programa se implementó por los profesores de los centros escolares y por psicólogos externos. Participaron un total de 3 centros de Educación Secundaria asignados aleatoriamente a las diferentes condiciones experimentales (grupo 1: programa Saluda aplicado por profesores, y grupo 2: programa Saluda aplicado por psicólogos externos) y a un grupo control en lista de espera. Se utilizó un diseño cuasi-experimental con mediciones pretest, postest y seguimiento (tras 12 meses de su aplicación). El grado de dificultad del programa se valoró según el número de sesiones, la estandarización en la estructura de las sesiones y la mayor o menor presencia de técnicas cognitivo-conductuales. Esto hizo considerar que se trataba de una intervención de complejidad media y, por esta razón se obtendrían resultados similares tanto si el programa era aplicado por psicólogos como por docentes del centro educativo. En los resultados de este estudio se hallaron menores incrementos de consumo de alcohol a corto plazo cuando el programa fue aplicado por docentes y de consumo de cannabis a largo plazo cuando fue aplicado por un psicólogo externo. En la evaluación de seguimiento el programa aplicado por docentes fue el que mayor tamaño del efecto alcanzó en todas las dimensiones. Asimismo, a pesar de que el programa Saluda no se diseñó específicamente para la prevención del consumo de tabaco, en los resultados de este estudio se obtuvo efectos positivos, en términos de reducción de consumo, cuando éste era aplicado por docentes. En síntesis, los docentes fueron los agentes más eficaces tras la implementación del programa Saluda. 
Efectos del programa sobre la actitud hacia las drogas

Espada, Hernández, Orgilés y Méndez (2010) presentaron un estudio en el que se analizó el efecto del programa Saluda sobre la actitud hacia las drogas en una muestra de adolescentes. Los participantes fueron estudiantes de tercer curso pertenecientes a un centro de Educación Secundaria. Los grupos (aulas de clase) fueron asignados aleatoriamente a una de las condiciones siguientes: programa Saluda, intervención placebo, intervención inespecífica (programa de Educación para la Salud), un programa de eficacia probada (Life Skills Training) y un grupo control que no recibió intervención. Se utilizó un diseño cuasi-experimental con mediciones pretest, postest y seguimiento (tras 3 y 6 meses de su aplicación postest).

Tras el análisis de la intención de consumo, y de la actitud hacia las drogas, el programa Saluda produjo más efectos que el resto de programas, para disminuir la intención de consumo de alcohol y promover actitudes favorables al no consumo de drogas. No se encontraron diferencias entre el grupo de Educación para la Salud y Atención Placebo para disminuir el consumo de alcohol. Finalmente, intervenir en cualquiera de sus modalidades fue más eficaz que no recibir algún tipo de intervención.

\section{Contribución de los componentes nucleares}

En el año 2012, Espada, Griffin, Pereira, García-Fernández y Orgilés publicaron el primer estudio de desmantelamiento parcial del programa con el objeto de analizar la contribución específica de los componentes de entrenamiento en habilidades sociales y de entrenamiento en resolución de problemas. Se pretendía comprobar la aportación de estos componentes, y optar por versiones más eficientes del programa. Los participantes fueron estudiantes pertenecientes a dos centros de Educación Secundaria. Los grupos (aulas de clase) fueron asignados aleatoriamente a una de las cuatro condiciones siguientes: programa completo, programa sin entrenamiento en resolución de problemas, programa sin entrenamiento en habilidades sociales y un grupo control que no recibió intervención. Se utilizó un diseño cuasiexperimental con mediciones pretest, postest y seguimiento (tras 12 meses de su aplicación). Los resultados de este estudio ponen de manifiesto la eficacia del programa Saluda completo frente al grupo que no recibió intervención en términos de menores tasas de consumo y cambios significativos de mejora en variables de riesgo. Además, el programa completo obtuvo mejores resultados que el programa sin uno de sus componentes. Por otro lado, efectos es- 
pecíficos fueron hallados en las dos versiones incompletas del programa. Las versiones incompletas sin habilidades sociales y sin entrenamiento en resolución de problemas producían cambios en estas habilidades. Sin embargo, el programa que incluyó habilidades sociales mostró mejores resultados en comparación con el grupo en lista de espera, pero no tanto como cuando se administró junto con el componente de entrenamiento en resolución de problemas (la condición programa Saluda completo). Estos hallazgos mostraron la existencia de un efecto aditivo entre el componente de habilidades sociales y de resolución de problemas, de tal modo que el déficit de uno de ellos tenía efectos en el otro para incidir sobre variables mediadoras y de resultado en el programa Saluda.

\section{Contribución de las tareas para casa}

Gonzálvez, Espada, Carballo, Orgilés y Piqueras (2012) analizaron si las tareas para casa del programa producían un efecto positivo en la autoeficacia, intención de consumo y percepción de riesgo. Los participantes fueron estudiantes pertenecientes a un centro de Educación Secundaria. Cada uno de los grupos (aulas de clase) fueron asignados aleatoriamente a una de las dos condiciones siguientes: programa estándar y programa sin actividades para casa. Mediante un diseño pretest-postest no se hallaron diferencias significativas entre los grupos tras la aplicación de los programas. Estos datos sugieren que los efectos de la intervención preventiva no disminuyen al no incluir tareas para casa.

\section{Contribución de los componentes secundarios}

Para el análisis de la contribución de los componentes compromiso público, resistencia a la publicidad y fomento del ocio saludable, participaron un total de 5 centros de Educación Secundaria asignados aleatoriamente a cuatro condiciones experimentales (programa Saluda completo, programa Saluda sin el componente de compromiso público, programa Saluda sin el componente de resistencia a la publicidad, programa Saluda sin el componente de fomento del ocio saludable) y un grupo control en lista de espera (Hernández, Griffin, GarcíaFernández, Orgilés y Espada, 2013). Se utilizó un diseño cuasi-experimental con mediciones pretest, postest y seguimiento (tras 6 meses de su aplicación). Todas las intervenciones resultaron más eficaces que la no-intervención para incidir sobre las variables protectoras y el número de episodios de embriaguez. Atendiendo a la eficacia de los tres componentes, al retirar el componente de promoción de ocio saludable, programa de Saluda no muestra ninguna 
pérdida de eficacia con respecto a la reducción de consumo de sustancias y otras variables de protección, mientras que el compromiso público y la resistencia a la publicidad mejoran la eficacia del programa antes mencionado. Por lo tanto, es posible mejorar el equilibrio entre costes de intervención mediante la implementación del programa con los componentes básicos y los componentes secundarios compromiso público y resistencia a la publicidad, y la exclusión de promoción del ocio saludable.

\section{Evaluación de una versión reducida}

Un último estudio de tipo exploratorio se llevó a cabo con el objetivo de evaluar los efectos de una nueva versión del programa Saluda en la que no se incluye el componente de fomento del ocio saludable y las tareas para casa (Hernández, Espada, Piqueras, Orgilés y García-Fernández, 2013). Esta versión reducida fue aplicada a una muestra de escolares de un centro de Educación Secundaria. Se trata de un estudio piloto con un diseño pre-post de un único grupo. Este estudio concluyó que una versión reducida del programa Saluda en la que se prescinde del componente fomento del ocio saludable y las tareas para casa presenta efectos positivos para reducir el porcentaje de episodios de embriaguez e incidir de forma significativa sobre las variables protectoras del consumo.

\section{Conclusiones}

En este trabajo se revisan los estudios controlados que evalúan el programa Saluda con el fin de analizar las evidencias a favor y determinar aspectos pendientes. Para ello se describe la trayectoria del programa a lo largo de doce años aportando evidencias sobre su eficacia preventiva. Tras la evaluación de una versión piloto del programa y cumplir con criterios de inclusión, Saluda fue incluido en el año 2004 en la base de datos EDDRA del European Monitoring Centre for Drugs and Drug Abuse (en su denominación en inglés "Weekend coming"), con una calificación de 2 en su nivel de calidad (una de las más altas que obtienen los programas españoles). A partir de aquí, se han desarrollado estudios de tipo longitudinal que han ofrecido datos cuantitativos sobre su eficacia (véase la tabla 2). 
Tabla 2. Estudios sobre la eficacia del programa Saluda Espada Sánchez, J. et al.

\begin{tabular}{|c|c|c|c|c|c|}
\hline Autor/es (año) & $\mathrm{N}$ & Edad & Grupos de intervención & Objetivo & Resultados \\
\hline $\begin{array}{l}\text { Espada, Orgilés, } \\
\text { Méndez, García- } \\
\text { Fernández e Inglés } \\
\text { (2008) }\end{array}$ & 133 & $13-15$ & $\begin{array}{l}\text { 1. PS } \\
\text { 2. Life Skills Training } \\
\text { 3. Educación para la Salud } \\
\text { 4. Atención-Placebo } \\
\text { 5. GC }\end{array}$ & $\begin{array}{l}\text { Valorar los efectos sobre factores } \\
\text { de riesgo cognitivos }\end{array}$ & $\begin{array}{l}\text { Se incrementa el nivel de información sobre } \\
\text { el alcohol y drogas sintéticas, y estimación } \\
\text { normativa }\end{array}$ \\
\hline $\begin{array}{l}\text { Pereira y García- } \\
\text { Fernández (2009) }\end{array}$ & 543 & $11-16$ & $\begin{array}{l}\text { 1. Adolescencia Temprana } \\
\text { 2. Adolescencia Media } \\
\text { 3. GC (Adolescencia Temprana) } \\
\text { 4. GC (Adolescencia Media) }\end{array}$ & $\begin{array}{l}\text { Determinar la eficacia según la } \\
\text { edad de los participantes }\end{array}$ & $\begin{array}{l}\text { Adolescencia temprana en intención y fre- } \\
\text { cuencia de consumo de alcohol, y norma } \\
\text { percibida }\end{array}$ \\
\hline $\begin{array}{l}\text { Espada, Griffin, Perei- } \\
\text { ra y García-Fernández } \\
(2010)\end{array}$ & 341 & $12-15$ & $\begin{array}{l}\text { 1. PS } \\
\text { 2. PS-EHS } \\
\text { 3. PS-ERP } \\
\text { 4. LE }\end{array}$ & $\begin{array}{l}\text { Analizar la contribución de los } \\
\text { componentes: entrenamiento en } \\
\text { habilidades sociales y resolución } \\
\text { problemas }\end{array}$ & $\begin{array}{l}\text { Impacto de los componentes en variables } \\
\text { mediadoras y de resultado. Efecto sinérgico. }\end{array}$ \\
\hline $\begin{array}{l}\text { Espada, Hernández, } \\
\text { Orgilés y Méndez } \\
(2010)\end{array}$ & 133 & $13-15$ & $\begin{array}{l}\text { 1. PS } \\
\text { 2. Life Skills Training } \\
\text { 3. Educación para la Salud } \\
\text { 4. Atención-Placebo } \\
\text { 5. GC }\end{array}$ & $\begin{array}{l}\text { Valorar los efectos sobre la acti- } \\
\text { tud hacia las drogas }\end{array}$ & $\begin{array}{l}\text { Disminuye la intención de consumo de al- } \\
\text { cohol y aumenta la actitud pro-salud frente } \\
\text { a las drogas }\end{array}$ \\
\hline Gázquez (2010) & 280 & $11-12$ & $\begin{array}{l}\text { 1. PS por docentes } \\
\text { 2. PS por psicólogos } \\
\text { 3. LE }\end{array}$ & $\begin{array}{l}\text { Determinar la eficacia según el } \\
\text { aplicador (docente/ psicólogo) }\end{array}$ & Docente $>$ Psicólogo \\
\hline $\begin{array}{l}\text { Gonzálvez, Espada, } \\
\text { Carballo, Orgilés y } \\
\text { Piqueras (2012) }\end{array}$ & 205 & $10-15$ & $\begin{array}{l}\text { 1. PS estándar } \\
\text { 2. PS con actividades comple- } \\
\text { mentarias }\end{array}$ & $\begin{array}{l}\text { Comprobar el efecto de la inclu- } \\
\text { sión de tareas para casa }\end{array}$ & $\begin{array}{l}\text { Prescindir de la inclusión de tareas para } \\
\text { casa no disminuye el efecto de la interven- } \\
\text { ción }\end{array}$ \\
\hline $\begin{array}{l}\text { Hernández, Espada, } \\
\text { Piqueras, Orgilés y } \\
\text { García-Fernández } \\
(2013)\end{array}$ & 106 & $14-17$ & 1. PS-(OS y AC) & $\begin{array}{l}\text { Evaluar los efectos del programa } \\
\text { sobre el consumo de drogas y } \\
\text { variables protectoras }\end{array}$ & $\begin{array}{l}\text { Reducción en el porcentaje de escolares que } \\
\text { presenta episodios de embriaguez, así como } \\
\text { un efecto de mejora significativa sobre las } \\
\text { variables protectoras del consumo }\end{array}$ \\
\hline $\begin{array}{l}\text { Hernández, Griffin, } \\
\text { García-Fernández, } \\
\text { Orgilés y Espada } \\
\text { (2013) }\end{array}$ & 567 & $14-16$ & $\begin{array}{l}\text { 1. PS } \\
\text { 2. PS-CP } \\
\text { 3. PS-RP } \\
\text { 4. PS-OS } \\
\text { 5. LE }\end{array}$ & $\begin{array}{l}\text { Analizar la contribución de los } \\
\text { componentes: compromiso públi- } \\
\text { co, análisis de la publicidad y } \\
\text { fomento del ocio saludable }\end{array}$ & $\begin{array}{l}\text { Sin fomento del ocio saludable puede mejo- } \\
\text { rar el balance coste/beneficio de la inter- } \\
\text { vención. }\end{array}$ \\
\hline
\end{tabular}

- 182 - Electronic Journal of Research in Educational Psychology, 13(1), 171-188. ISSN: 1696-2095. 2015, no. 35 http://dx.doi.org/10.14204/ejrep.35.14052 
Los resultados de estos estudios constatan la eficacia del programa para mejorar las capacidades cognitivas de protección frente al consumo de drogas incrementando el nivel de información sobre el alcohol y las drogas de síntesis, así como la estimación normativa sobre el consumo. Tras valorar los efectos sobre la actitud hacia las drogas, el programa disminuye la intención de consumo de alcohol y aumenta la actitud pro-salud frente a las drogas. Este último resultado se encuentra en consonancia con los datos hallados en un reciente metaanálisis que proporciona evidencia sobre la eficacia de los programas preventivos escolares del abuso de drogas en España para modificar las actitudes hacia las drogas (Espada et al., 2015).

El programa ha mostrado mayores efectos en la adolescencia temprana para reducir el consumo de alcohol, datos que encuentran apoyo en los resultados de otros trabajos (Chassin, Pitts y Prost, 2002; Gómez-Fraguela, Luengo, y Romero, 2002; Gottfredson y Wilson, 2003; Oliva, Parra y Sánchez-Queija, 2008; Resnicow y Botvin, 1993). Atendiendo a los efectos positivos del programa sobre la adolescencia temprana frente a la adolescencia media, un futuro reto consistiría en analizar los efectos sobre la adolescencia tardía y observar así los posibles cambios sobre las drogas sintéticas por ser éstas las más prevalentes en su consumo a edades más avanzadas (entre los 17-18 años) teniendo en cuenta los datos más recientes del OEDT (2013).

Respecto al responsable de la implementación del programa, el docente fue el agente más eficaz mejorando e influyendo sobre la mayoría de las variables. En esta línea, otros trabajos destacan el descenso en el consumo cuando es el profesor quien aplica el programa (Alonso y del Barrio, 1997; Bruvold, 1993). Sin embargo, otros estudios destacan la importancia de la implementación de los programas por profesionales y profesorado de forma conjunta (Espada et al., 2015). Pese a que en la actualidad no existe consenso en cuanto al tipo de aplicador más apropiado, lo que sí queda claro es el papel fundamental que juega como moderador de los efectos del programa (Gottfredson y Wilson, 2003). En este sentido, sería conveniente llevar a cabo estudios donde se evalúen las características específicas de los propios evaluadores con el fin de detectar qué competencias son las más idóneas para la implementación de programas preventivos de drogodependencias (Cuijpers, 2002).

En referencia al consumo de alcohol, los datos indicaron una reducción del número de participantes que presentaron episodios de embriaguez (atracones). Este resultado se encuen- 
tra justificado por la especificidad del programa Saluda para incidir sobre el consumo abusivo. No obstante, teniendo en cuenta que la mayor parte de los estudios realizados acerca del programa Saluda presentan seguimientos de tres, seis, y doce meses, sería conveniente replicar estos estudios con períodos de seguimiento más largos para observar cambios en la incidencia y frecuencia del consumo.

Asimismo, estudios recientes sobre el análisis de los componentes del programa Salu$d a$ revelan un mayor impacto del programa cuando éste incluye el entrenamiento en habilidades sociales y la habilidad para resolver problemas, además de un efecto sumatorio. Sin embargo, otros estudios sobre esta última habilidad apuntan a la necesidad de clarificar qué factores determinan la existencia o no existencia de relaciones entre la habilidad para resolver problemas y el no consumo de drogas en la etapa adolescente (Hernández, Espada y GuillénRiquelme, en prensa). Por otro lado, cuando se eliminó el componente de fomento del ocio saludable, el programa no pierde eficacia en términos de reducción del consumo de sustancias y otras variables de riesgo, y los componentes de compromiso público y resistencia a la publicidad lo mejoran. Por tanto, partiendo de los resultados positivos hallados en este estudio sobre el componente compromiso público y la resistencia a la publicidad, junto a otros trabajos que apoyan su importancia (Cuijpers, 2002; González, Gómez-Durán y García, 2000; Hansen, 1992; Hansen, Dusenbury, Bishop y Derzon, 2007; Precioso y Macedo, 2003; Skara y Sussman, 2003), se sugiere otorgar un papel más relevante a estos componentes considerando los efectos sustanciales que proporcionan sobre variables de consumo y protectoras. Al mismo tiempo, se propone suprimir el componente fomento del ocio saludable por no considerarse óptimo en términos de coste-beneficio. Las tareas para casa pasarían a tener carácter opcional, teniendo en cuenta que la no inclusión de estas tareas en el programa Saluda no merma sus efectos positivos.

A partir de las evidencias de los estudios controlados, se puede concluir que el programa Saluda cuenta con resultados cuantitativos de eficacia sobre variables de consumo y protectoras. Además se dispone de datos sobre los mejores parámetros para su aplicación, como el agente de implementación más eficaz, la edad de los destinatarios más apta para intervenir y los contenidos específicos que mejoran el balance coste/resultados de la intervención. Continúan siendo necesarios estudios de seguimiento más prolongados con el fin de comprobar el mantenimiento de estos cambios y la reducción de conductas de consumo, así como realizar estudios experimentales, con asignación aleatoria de los participantes, para in- 
crementar la validez a los resultados de los estudios tal como indican otros trabajos de investigación (Jiménez et al., 2014).

\section{Referencias}

Alonso, C. y Del Barrio, V. (1997). Efectividad de tres intervenciones para la prevención del consumo de tabaco en el medio escolar. Revista de Psicología General y Aplicada, 50 (2), 223-241.

Botvin, G. J. (1998). Life Skills Training. A drug and violence prevention program. New York: Institute for Prevention Research, Cornell University Medical College.

Brown, S. (2008). Developmental perspective on alcohol and youths 16 to 20 years of age. Pediatrics, 121, 290-310. doi:10.1542/peds.2007-2243D

Bruvold, W. H. (1993). A meta-analysis of adolescent smoking prevention programs. American Journal of Public Health, 83, 872-880. doi:10.2105/AJPH.83.6.872

Canning, U., Millward, L., Raj, T. y Warm, D. (2004). Drug use prevention among young people: a review of reviews. London: Health Development Agency.

Chassin, L., Pitts, S. C. y Prost, J. (2002). Binge drinking trajectories from adolescence to emerging adulthood in a high-risk sample: predictors and substance abuse outcomes. Journal of Consulting and Clinical Psychology, 70, 67-78. doi:10.1037//0022006X.70.1.67

Cuijpers, P. (2002). Effective ingredients of school-based drug prevention programs. A systematic review. Addictive Behaviors, 27, 1009-1023. doi:10.1016/S03064603(02)00295-2

European Monitoring Centre for Drugs and Drug Addiction (2009). Evidence based information on universal prevention in non-school setting-an overview. http://www.emcdda.europa.eu/themes/best-practice/evidence. Extraído el 21 de Febrero de 2015.

Espada, J. P. y Méndez, F. X. (2003). Prevención del abuso de alcohol y drogas de síntesis. Madrid: Pirámide.

Espada, J. P., Gonzálvez, M. T., Orgilés, M., Lloret, D. y Guillén-Riquelme, A. (2015). Metaanalysis of the effectiveness of school substance abuse prevention programs. Psicothema, 27(1), 5-12. doi: 10.7334/psicothema2014.106

Espada, J. P., Griffin, K. W., Pereira, J. R., Orgilés, M. y García-Fernández, J. M. (2012). Component analysis of a school-based substance use prevention program in Spain: 
Contributions of problem solving and social skills training content. Prevention Science, 13, 86-95. doi:10.1007/s11121-011-0249-y

Espada, J. P., Hernández, O., Orgilés, M. y Méndez, F. X. (2010). Comparación de distintas estrategias para la modificación de la actitud hacia el consumo de drogas en escolares. Electronic Journal of Research in Educational Psychology, 8(3), 315-328.

Espada, J. P., Méndez, F. X., Botvin, G. J., Griffin, K. W., Orgilés, M. y Rosa, A. I. (2002). ¿Éxito o fracaso de la prevención del abuso de drogas en el contexto escolar? Un metaanálisis de los programas en España. Psicología Conductual, 10(3), 581-602.

Espada, J. P., Orgilés, M., Méndez, F. X., García-Fernández, J. M. e Inglés, C. (2008). Efectos del programa Saluda sobre factores cognitivos relacionados con el consumo de drogas. Salud y Drogas, 8(1), 29-50.

Espada, J.P., Rosa, A. I. y Méndez, F. X. (2003). Eficacia de los programas de prevención escolar con metodología interactiva. Salud y Drogas, 3(2), 61-82.

Faggiano, F., Vigna-Taglianti, F. D., Versino, E., et al. (2008). School-based prevention for illicit drugs use: A systematic review. Preventive Medicine, 46, 385-396. doi:10.1016/j.ypmed.2007.11.012

Gázquez, M. (2010). Comparación de tres programas de prevención de drogas en el ámbito escolar. Tesis doctoral. Universidad Miguel Hernández.

Gázquez, M., García, J. A. y Espada, J. P. (2009). Características de los programas eficaces para la prevención escolar del consumo de drogas. Health and Addictions, 9, 185-208.

González, M., Fernández, M. E., Urturi, A., Moreno, M. F., Molinero, L., Gutiérrez, P. (2014). Consumo y actitud ante el alcohol de los adolescentes de 13-18 años de edad en la provincia de Valladolid. Acta Pediátrica Española, 72(6), 111-118.

Gonzálvez, M. T., Espada, J. P., Carballo, J. L., Orgilés, M. y Piqueras, J. A. (2012). Contribución de las tareas para casa en un programa preventivo. XXXIX Jornadas Nacionales Socidrogalcohol. Tarragona.

Gómez-Fraguela, J. A., Luengo, M. A. y Romero, E. (2002). Prevención del consumo de drogas en la escuela: cuatro años de seguimiento de un programa. Psicothema, 14(4), 685692.

González, S., Gómez-Durán, B. y García, M. P. (2000). Prevención del tabaquismo: Evaluación de la eficacia de un programa educativo-preventivo. Revista de Psicología General y Aplicada, 53, 661-671. 
Gottfredson, D. C. y Wilson, D. B. (2003). Characteristics of effective school-based substance abuse prevention. Prevention Science, 4(1), 27-38. doi: 10.1023/A:1021782710278

Hansen, W. B. (1992). School-based substance abuse prevention: A review of the state of the art in curriculum: 1980-1990. Health Educational Research, 7(3), 403-430. doi: 10.1093/her/7.3.403

Hansen, W. B, Dusenbury, L., Bishop D. y Derzon, J. H. (2007). Substance abuse prevention program content: systematizing the classification of what programs target for change. Health Education Research, 22(3), 351-360. doi:10.1093/her/cyl091

Hernández, O., Espada, J. P. y Guillén-Riquelme, A. (en prensa). Relación entre conducta prosocial, resolución de problemas y consumo de drogas en adolescentes. Anales de Psicología.

Hernández, O., Espada, J. P., Piqueras, J. A., Orgilés, M. y García-Fernández, J. M. (2013). Programa de prevención del consumo de drogas Saluda: Evaluación de una nueva versión en adolescentes españoles. Health and Addictions, 13(2), 135-144.

Hernández, O., Griffin, K. W., García-Fernández, J. M., Orgilés, M. y Espada, J. P. (2013). Public commitment, resistance to advertising, and leisure promotion in a school-based drug abuse prevention program: A component dismantling study. Journal of Drug Education, 43(4), 331-351. doi:10.2190/DE.43.4.c

Hunter, L. y Elias, M. J. (2000). Interracial friendships, multicultural sensitivity, and social competence: How are they related?. Journal of Applied Developmental Psychology, 20(4), 551-573. doi:10.1016/S0193-3973(99)00028-3

Jiménez, L., Antolín-Suárez, L., Oliva, A., Hidalgo, V., Jiménez-Iglesias, A., Lorenze, B., Moreno, C. y Ramos, P. (2014). A synthesis of research on effectiveness of schoolbased drug prevention programmes. Culture and Education, 26(3), 573-602. doi: $10.1080 / 11356405.2014 .973670$

Karki, S., Pietila, A. M., Lansimies-Antikainen, H., Vajoranta, P., Pirskanen, M. y Laukanen, E. (2012). The effects of interventions to prevent substance use among adolescents: A systematic review. Journal of Child \& Adolescent Substance Abuse, 21(5), 383-413. doi:10.1080/1067828X.2012.724276

Masten, A. y Faden, V. (2008). Underage drinking: A developmental framework. Pediatrics, 121, 235-251. doi:10.1542/peds.2007-2243A 
Miller, J., Naimi, T., Brewer, R. y Everett, S. (2007). Binge drinking and associated health risk behaviors among high school students. Pediatrics, 119, 76-85. doi: 10.1542/peds.2006-1517

Observatorio Español de la Droga y las Toxicomanías (2013). Encuesta estatal sobre uso de drogas en estudiantes de enseñanza secundaria (ESTUDES, 2012/2013). Madrid: Delegación del Gobierno para el Plan Nacional Sobre Drogas, Ministerio del Interior.

Oliva, A., Parra, A. y Sánchez-Queija, I. (2008). Consumo de sustancias durante la adolescencia: trayectorias evolutivas y consecuencias para el ajuste psicológico. International Journal of Clinical and Health Psychology, 8(1), 153-169.

Pereira, J. R. y García-Fernández, J. M. (2009). Evaluación de los efectos del programa preventivo Saluda según la edad de los participantes. Salud y Drogas, 9(1), 93-111.

Precioso, J. y Macedo, M. (2003). La contribución de los programas de influencias psicosociales (complementados con otras estrategias) en el control de la epidemia tabáquica. Adicciones, 15(4), 369-378.

Resnicow, K. y Botvin, G. J. (1993). School-based substance use prevention programs: Why do effects decay? Preventive Medicine: An International Journal Devoted to Practice and Theory, 22, 484-490. doi:10.1006/pmed.1993.1039

Skara, S. y Sussman, S. (2003). A review of 25 long-term adolescent tobacco and other drug use prevention program evaluations. Preventive Medicine, 37, 451-474. doi:10.1016/S0091-7435(03)00166-X

Sussman, S., Unger, J. B. y Dent, C. W. (2004). Peer group self-identification among alternative high school youth: A predictor of their psychosocial functioning five years later. International Journal of Clinical and Health Psychology, 4, 9-25.

Tobler, N. S., Roona, M. R., Ochshorn, P., Marshall, D. G., Streke, A. V. y Stackpole, K. M. (2000). School-based adolescent drug prevention programs: 1998 meta-analysis. Journal of Primary Prevention, 20(4), 275-336. doi:10.1023/A:1021314704811 